\title{
Lobar Radioembolization for Intermediate and Advanced Hepatocellular Carcinoma: Retrospective and Prospective Data
}

\author{
Janesh Lakhoo, MD, MS ${ }^{1}$ Thomas H. Perez, BS ${ }^{1}$ Anthony J. Borgmann, MD ${ }^{1}$ Daniel B. Brown, MD ${ }^{1}$ \\ ${ }^{1}$ Department of Radiology, Vanderbilt University Medical Center, \\ Nashville, Tennessee \\ Address for correspondence Daniel B. Brown, MD, Department of \\ Radiology, Vanderbilt University Medical Center, 1161 21st Avenue S, \\ CCC-1118 Medical Center North, Nashville, TN 37232 \\ Semin Intervent Radiol 2021;38:412-418 \\ (e-mail: daniel.b.brown@vumc.org).
}
Abstract
Keywords
- hepatocellular carcinoma
- Barcelona Clinic Liver Cancer
- radioembolization
- chemoembolization
- interventional radiology

Historically, outcomes reporting for radioembolization with yttrium-90 $\left({ }^{90} \mathrm{Y}\right)$ of hepatocellular carcinoma has included patients across the range of Barcelona Clinic Liver Cancer (BCLC) stages. With the potential for curative radiation segmentectomy for BCLC 0/A patients and evolution of systemic therapy for BCLC C patients, focused review by group is of increasing interest. In this review, we report on efficacy of ${ }^{90} \mathrm{Y}$ in patients with intermediate (BCLC B) and advanced (BCLC C) hepatocellular carcinoma as well as expected toxicities. Additionally, we review existing trials comparing ${ }^{90} \mathrm{Y}$ to transarterial chemoembolization and systemic treatments in these patient groups and outline future studies.
Hepatocellular carcinoma (HCC) is the sixth most common cancer, accounts for the majority of primary liver cancers, and is the fourth leading cause of cancer-related deaths worldwide. ${ }^{1}$ Patients who present with liver cancer are staged according to the Barcelona Clinic Liver Cancer (BCLC) staging system that is determined by the patient's performance status, Child-Pugh (CP) score, and radiologic tumor extent (size, number, metastases, and vascular invasion). The classification system categorizes patients into five groups ( $0, A, B, C$, and $D)$ which helps guide management. BCLC 0 patients (tumor $<2 \mathrm{~cm}$ in size, Eastern Cooperative Oncology Group [ECOG] 0, Child-Pugh A) may potentially be cured with resection, ablation, or radiation segmentectomy. BCLC A (one to three tumors less than $3 \mathrm{~cm}$, ECOG 0, ChildPugh A-B) may potentially undergo transplantation. Unfortunately, most patients present with intermediate (BCLC B; multinodular, unresectable) or advanced (BCLC C; vascular invasion and/or impaired performance status) disease and are not candidates for curative interventions at the time of diagnosis. ${ }^{2}$ This review will provide an overview of ${ }^{90} \mathrm{Y}$ therapy for intermediate and advanced stage HCC (BCLC B and $\mathrm{C}$ ), including its outcomes, its safety, and additional research being performed.

\section{Treatment Options}

Patients beyond the Milan criteria ( 1 tumor $\leq 5 \mathrm{~cm}, \leq 3$ tumors $\leq 3 \mathrm{~cm}$ ) may be considered for downstaging if they are within the University of California San Francisco criteria (1 tumor $\leq 6.5 \mathrm{~cm}$ or $\leq 3$ tumors with maximal diameter $<4.5 \mathrm{~cm}$ and total diameter $<8 \mathrm{~cm}$ ). The remaining BCLC $\mathrm{B}$ and $C$ patients are treated palliatively. Locoregional therapy is commonly the first-line treatment for nontransplantable BCLC B patients. Y90 has similar survival benefit with significantly longer time to progression when compared to transarterial chemoembolization (TACE). Additionally, Y90 provides superior quality of life compared to TACE in patients with intermediate and advanced $\mathrm{HCC}^{3,4}{ }^{90} \mathrm{Y}$ has superseded TACE in some practices as the primary treatment for these patients. $^{5}$

While systemic therapy is increasingly performed in patients with BCLC C disease, locoregional therapy can play
Issue Theme Seminars in Radioembolization; Guest Editors, Robert J. Lewandowski, MD, FSIR and William Rilling, MD (c) 2021. Thieme. All rights reserved.

Thieme Medical Publishers, Inc., 333 Seventh Avenue, 18th Floor, New York, NY 10001, USA
DOI https://doi.org/ 10.1055/s-0041-1733903. ISSN 0739-9529. 
a role in patient care, especially in patients who do not tolerate systemic therapy. Patients with BCLC D tumors (extrahepatic disease) are usually treated with systemic therapy unless there are symptoms from distension of the liver capsule from bulky disease.

\section{Outcomes}

Patient response and survival following ${ }^{90} \mathrm{Y}$ is linked to multiple factors including BCLC stage, portal vein thrombosis (PVT), performance status, previous arterial and/or systemic therapy, and unilobar versus bilobar disease. ${ }^{6,7}$ Patients with earlier stage disease have longer overall survival (OS), though ${ }^{90} \mathrm{Y}$ still provides benefit in those with intermediate and advanced stage disease (-Fig. 1). In their study, Mantry et al found that those patients with early-stage disease (BCLC A) had an OS of 27.8 months (95\% confidence interval [CI]: 12.935.3 months) compared to 11.4 (95\% CI: 8.3-16.7) and 9.2 (95\% CI: 3.6-17.2) months for those with intermediate (BCLC B) and advanced disease (BCLC C). ${ }^{6}$ Similarly, a recent study by Frantz et al evaluating 448 patients who were primarily palliative (only 22\% were within the Milan criteria) treated with resin ${ }^{90} \mathrm{Y}$ demonstrated the best survival with earlystage disease (median OS rates for BCLC A, B, C, and D were $>30$ months, $19.5,13.6$, and 11.5 months, respectively). ${ }^{8}$

Ali et al found a discrepancy in OS between BCLC C patients with ECOG PS 1 compared to patients with PVT or ECOG PS2. In their study, ECOG PS 1 patients had a median survival of 19.4 months compared to 7.7 months for those presenting with PVT or ECOG PS 2, suggesting that ECOG PS1 as an isolated variable is a poor indicator of advanced disease. ${ }^{7}$ Mazzaferro et al also found the median OS to be 15 months (95\% CI: 12-18 months) in BCLC B and C patients treated with ${ }^{90} \mathrm{Y}$. BCLC C patients without portal vein thrombus had increased survival compared to those with venous occlusion (18 vs. 13 months) which did not reach statistical significance. $^{9}$

With greater availability of cone beam computed tomography (CT), complete tumor coverage has improved and activity of prescribed ${ }^{90} \mathrm{Y}$ more closely reflects the tumor volume and perfusion compared to surrounding parenchyma. Allimant et al used PET/CT following radioembolization in 38 patients to determine accuracy of tumor targeting and coverage in 42 treatments. They found that overall and progression-free survival (PFS) rates were lower in patients with incomplete tumor targeting (median OS of 4.5 and PFS 2.7 months, $p<0.001$ ) compared to those who had complete tumor targeting (median OS of 19.2 and PFS of 7.9 months, $p<0.001) .{ }^{10}$ Patients with incomplete targeting (17/42) all had progression of disease within 6 months with no difference between BCLC B and C patients (odds ratio [OR]: 0.72, 95\% CI: $0.23-2.387, p=0.489$ ).

A recent multicenter randomized phase II study (DOSISPHERE-01) compared standard ${ }^{90} \mathrm{Y}$ dosimetry to personalized dosimetry in patients with at least one tumor $\geq 7 \mathrm{~cm}$ unresectable HCC in patients receiving glass microspheres. The therapy goal was $205 \mathrm{~Gy}$ in the personalized group compared to $120 \mathrm{~Gy}$ in the standard group. Patients who were treated using personalized dosimetry had nearly double the objective response rate ( 70 vs. $36 \%, p=0.007$ ) and a significantly longer OS (26.6 vs. 10.7 months, $p=0.0096$ ) than patients treated with standard dosimetry. ${ }^{11}$ These
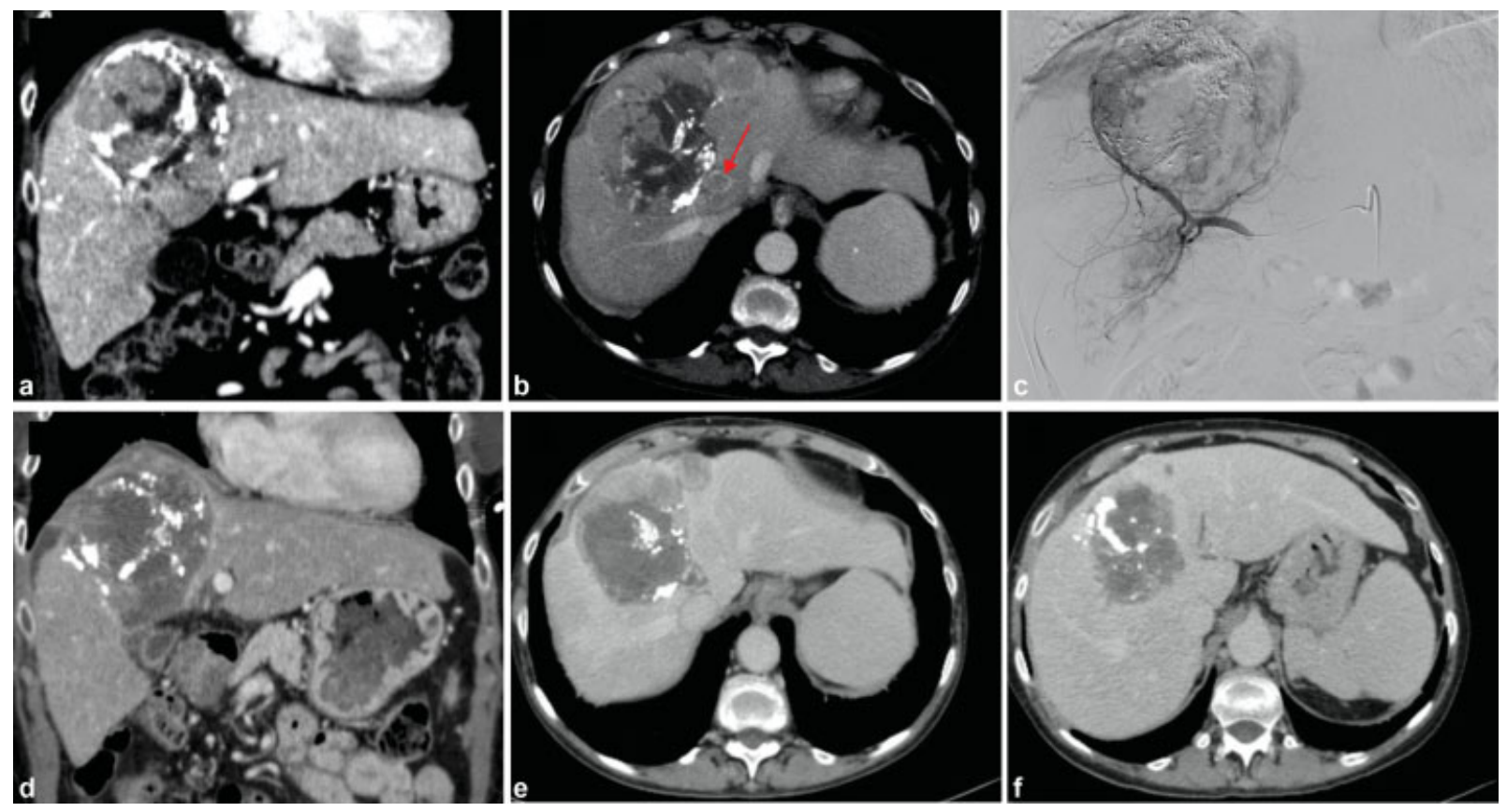

Fig. $1{ }^{90} \mathrm{Y}$ therapy for viable tumor following transarterial chemoembolization. Child-Pugh B patient with large, enhancing segment VIII mass with associated middle hepatic vein thrombus (red arrow) (BCLC C) on coronal (a) and axial (b) postcontrast CT measuring $13.2 \mathrm{~cm}$ in widest diameter, and demonstrating persistent enhancing viable tumor after treatment with chemoembolization. After radioembolization to the right anterior segmental artery (c), coronal (d) and axial (e, f) postcontrast CT images demonstrate extensive central necrosis and decreased enhancement. 
Table 1 Published outcomes focused on BCLC B and C hepatocellular carcinoma

\begin{tabular}{|c|c|c|c|c|}
\hline Author & Patient cohort & Type of ${ }^{90} \mathrm{Y}$ & $\mathrm{BCLC}$ & Median overall survival (month) \\
\hline Salem et al ${ }^{5}$ & 348 Child-Pugh A BCLC B/C & Glass & $\begin{array}{l}\text { BCLC B: } 91 \\
\text { BCLC C: } 257\end{array}$ & $\begin{array}{l}\text { BCLC B, Child Pugh A: } 25 \\
\text { BCLC C, Child Pugh A: } 15\end{array}$ \\
\hline Frantz et al ${ }^{8}$ & 151 Child-Pugh A BCLC B/C & Resin & $\begin{array}{l}\text { BCLC B: } 132 \\
\text { BCLC C: } 19\end{array}$ & $\begin{array}{l}\text { BCLC B, Child Pugh A: } 21.5 \\
\text { BCLC C, Child Pugh A: } 21.8\end{array}$ \\
\hline Mantry et al ${ }^{6}$ & $29 \mathrm{BCLC} B$ and $\mathrm{C}$ & Resin & $\begin{array}{l}\text { B: } 26 \\
\text { C: } 3\end{array}$ & $\begin{array}{l}\text { BCLC B: } 11.4 \\
\text { BCLC C: } 9.2\end{array}$ \\
\hline \multirow[t]{3}{*}{ Ali et al ${ }^{7}$} & 547 patients all BCLC C & Glass & BCLC C: 547 & Whole group: 10.7 \\
\hline & & & ECOG C: 233 & ECOG BCLC C: 19.4 \\
\hline & & & PVT C: 314 & PVT BCLC C: $7.7(p<0.0001)$ \\
\hline
\end{tabular}

Abbreviations: BCLC, Barcelona Clinic Liver Cancer; ECOG, Eastern Cooperative Oncology Group; PVT, portal vein thrombosis.

studies demonstrate that technique, dosimetry, and adequate coverage play an important role in outcomes (-Table 1).

\section{${ }^{90} \mathrm{Y}$ versus TACE}

There are no sizable prospective trials comparing efficacy of ${ }^{90} \mathrm{Y}$ and TACE in patients with BCLC B and C diseases; however, data in BCLC A disease suggest benefit of ${ }^{90} \mathrm{Y}$ over TACE. In the phase II PREMIER trial, Salem et al found that HCC patients, 35 of 45 patients who had BCLC A disease (78\%), treated with radioembolization had a significantly prolonged time to progression compared to TACE (>26 vs. 6.8 months, $p=0.0012$ ), which was the primary endpoint of that trial. $^{12,13}$ There was, however, no significant difference in median survival (18.6 vs. 17.7 months, $p=0.99$ ). This prospective study followed a retrospective review that also found no significant difference in OS between 123 patients treated with Y90 (17.4 months) and 122 patients treated with TACE (20.5 months, $p=0.2$ ). The BCLC B subgroup patients in this trial had almost identical OS: the $65^{90} \mathrm{Y}$ patients had an OS of 17.2 months compared to the 61 TACE patients surviving 17.5 months $(p=0.4) .{ }^{13}$ Patients receiving ${ }^{90} \mathrm{Y}$ had significantly less frequent abdominal pain $(p<0.001)$ and transaminase elevations $(p=0.004)$ than those undergoing TACE.

In a meta-analysis by Zhang et al evaluating 947 patients with intermediate or advanced HCC treated with ${ }^{90} \mathrm{Y}$ or TACE, OS of ${ }^{90} \mathrm{Y}$ patients was significantly greater than TACE, with a $26 \%$ reduction in death (hazard ratio: $0.74,95 \% \mathrm{CI}: 0.61-0.90$, $p<0.01$ ). While there was no significant difference in 1- or 2year OS rates, 3-year OS rates for ${ }^{90} Y$ were significantly higher than those treated with TACE (relative risk: $1.75,95 \% \mathrm{CI}$ : $1.01-3.03, p=0.05)$. This finding implies that while ${ }^{90} \mathrm{Y}$ may not impact immediate survival, the effects of radioembolization may enhance over time. This analysis also corroborated the findings of Salem et al, finding that the risk of progression after ${ }^{90} \mathrm{Y}$ was significantly less compared to HCC treated with TACE $(\mathrm{HR}=0.61,95 \% \mathrm{CI}: 0.41-0.89$, $p=0.01)$. Other findings included decreased hospitalization time due to multiple required cycles of TACE, (mean difference $=-2.66$ days, 95\% CI: 4.08-1.24, $p<0.01)$ and less abdominal pain $(\mathrm{RR}=0.30,95 \% \mathrm{CI}: 0.11-0.83, p=0.02) .{ }^{14}$

\section{${ }^{90} \mathrm{Y}$ versus Sorafenib}

The phase III SARAH trial ( - Table 2 ) by Vilgrain et al compared sorafenib to ${ }^{90} \mathrm{Y}$ in 467 patients after unsuccessful

Table 2 Outcomes of ${ }^{90} \mathrm{Y}$ alone or combined with sorafenib versus sorafenib monotherapy

\begin{tabular}{|c|c|c|c|c|}
\hline Author & Patient cohort & Type of ${ }^{90} \mathrm{Y}$ & $\mathrm{BCLC}$ & Median OS in month \\
\hline $\begin{array}{l}\text { Vilgrain et al } \\
\text { (SARAH) }\end{array}$ & $\begin{array}{l}467 \text { patients } \\
237 \text { Y90 } \\
222 \text { sorafenib }\end{array}$ & Resin & $\begin{array}{l}{ }^{90} Y: \\
\text { BCLC B: } 66 \\
\text { BCLC C: } 162 \\
\text { Sorafenib: } \\
\text { BCLC B: } 61 \\
\text { BCLC C: } 149\end{array}$ & $\begin{array}{l}{ }^{90} Y: 8 \\
\text { Sorafenib: } 9.9 \\
p=0.18\end{array}$ \\
\hline Chow et al $^{16}$ (SIRveNIB) & $\begin{array}{l}360 \text { patients } \\
182 \text { Y90 } \\
178 \text { sorafenib }\end{array}$ & Resin & $\begin{array}{l}{ }^{90} Y: \\
\text { BCLC B: } 93 \\
\text { BCLC C: } 88 \\
\text { Sorafenib } \\
\text { BCLC B: } 97 \\
\text { BCLC C: } 80\end{array}$ & $\begin{array}{l}{ }^{90} Y: 8.8 \\
\text { Sorafenib: } 10.0 \\
p=0.36\end{array}$ \\
\hline Ricke et al $^{17}$ (SORAMIC) & $\begin{array}{l}424 \text { patients } \\
216 \text { Y } 90+\text { sorafenib } \\
208 \text { sorafenib }\end{array}$ & Resin & All BCLC B or greater & $\begin{array}{l}\text { Combined }{ }^{90} \mathrm{Y} \text { and Sorafenib: } 12.1 \\
\text { Sorafenib: } 11.4 \\
p=0.9529\end{array}$ \\
\hline
\end{tabular}

Abbreviations: BCLC, Barcelona Clinic Liver Cancer; OS, overall survival. 
Table 3 Active and recruiting studies evaluating efficacy and outcomes of ${ }^{90} \mathrm{Y}$ in combination with other treatments

\begin{tabular}{|l|l|l|l|l|}
\hline Study arms & Patient population & Study phase/size & Device & Study identifier \\
\hline${ }^{90} \mathrm{Y}+$ SBRT & BCLC B and C & Single arm, Phase I & Glass & NCT04518748 \\
\hline${ }^{90} \mathrm{Y}+$ sorafenib & BCLC C & Single arm, Phase II & Glass & NCT01900002 \\
\hline $\begin{array}{l}90 \\
\text { Apatinib }\end{array}$ & BCLC C and PVT & $\begin{array}{l}\text { Randomized } \\
\text { controlled study }\end{array}$ & Not specified & NCT03520257 \\
\hline${ }^{90} \mathrm{Y}+$ pembrolizumab & Unresectable HCC; Child-Pugh A or B7 & Single-arm pilot study & Not specified & NCT03099564 \\
\hline${ }^{90} \mathrm{Y}+$ nivolumab & Unresectable HCC; Child-Pugh A or B7-8 & Single arm, Phase I & Glass & NCT03812562 \\
\hline${ }^{90} \mathrm{Y}+$ durvalumab & Unresectable HCC; Child-Pugh A or B7 & Single arm & Not specified & NCT04124991 \\
\hline
\end{tabular}

Abbreviations: BCLC, Barcelona Clinic Liver Cancer; HCC, hepatocellular carcinoma; NCT, National Clinical Trial; PVT, portal vein thrombosis; SBRT, stereotactic body radiation therapy.

TACE. BCLC B and C patients made up 439 of the 467 (94\%) enrolled group. There was no significant difference in median OS (9.9 months sorafenib; 8 months ${ }^{90} Y ; p=0.18$ ). Despite the lack of survival benefit, the radioembolization group showed a significantly better tumor response rate, better quality of life, and fewer toxicities. ${ }^{15}$

The phase III SIRveNIB multicenter trial by Chow et al evaluated 360 BCLC B and C patients from 11 Asia-Pacific countries. This study reported a similar tumor response rate in patients treated with ${ }^{90} \mathrm{Y}$ along with fewer adverse events compared to those treated with sorafenib. ${ }^{16}$ While the ${ }^{90} \mathrm{Y}$ group did not have survival benefit ( 8.8 vs 10.0 months of OS, $p=0.36$ ), radioembolization remains an option for patients with BCLC C HCC who cannot tolerate sorafenib or other systemic therapy.

Both SARAH and SIRveNIB measured OS on an intent-totreat basis. A number of patients enrolled to receive ${ }^{90} \mathrm{Y}$ did not undergo treatment in each trial: 63 of 237 (27\%) in SARAH and 52 of $182(28.6 \%)$ in SIRveNIB. Had all enrolled patients had therapy follow-through, there is a possibility that the outcomes may have been positive for ${ }^{90} \mathrm{Y}$. Many of the interventional radiologists in these trials were inexperienced ${ }^{90} Y$ users. These studies demonstrate the value that interventional radiology operators experienced with radioembolization or other technically demanding devices provide.

SORAMIC, a randomized controlled trial with 424 patients receiving either ${ }^{90} \mathrm{Y}$ therapy with resin microspheres and sorafenib versus sorafenib alone found no added benefit of combined therapy on median OS (12.1 vs. 11.4 months, $p=0.9529)$. Notably, there were significantly increased adverse events in the ${ }^{90} \mathrm{Y}+$ sorafenib group compared to the sorafenib alone group. ${ }^{17}$

\section{Y90 and Immunotherapeutic Agents}

Checkpoint inhibitor immunotherapy has improved survival in advanced hepatocellular carcinoma. ${ }^{18,19}$ The CheckMate 040 study $^{20}$ evaluating nivolumab and the KEYNOTE-224 ${ }^{21}$ study evaluating pembrolizumab have both shown objective responses in patients with advanced HCC. A study by Zhan et al found the combination of radioembolization with checkpoint inhibitors to be safe with limited treatment- related toxicities. There were no early mortality or grades $3 / 4$ hepatobiliary or immunotherapy-related toxicities within 30 days $^{19}$ and only two of the 26 studied patients developed grades $3 / 4$ toxicity in 1 - to 3 -month range in the setting of HCC disease progression. The median OS from first immunotherapy was 17.2 months (95\% CI, 6.6-26.4), and the median OS from first radioembolization was 16.5 months $(95 \% \mathrm{CI}, 4.2-7.2) .^{19}$ There are multiple prospective trials being performed evaluating the optimal combination treatment protocol and safety of ${ }^{90} \mathrm{Y}$ and immune checkpoint inhibitor immunotherapy ( - Table 3 ).

\section{Toxicity with ${ }^{90} \mathbf{Y}$}

Brown et al reviewed 6-month toxicities in 199 HCC patients who underwent resin microsphere therapy. ${ }^{22}$ This group included 32 patients with PVT and 104 patients with ECOG scores of 1 or greater. This patient group was heavily pretreated, including resection $(n=8,4.1 \%)$, chemoembolization $(n=50,25.4 \%)$, sorafenib $(n=28,14.2 \%)$, and ablation ( $n=24,12.2 \%)$. Toxicities were assessed using the common terminology criteria for adverse events (CTCAE) version 5 . At 6 months after treatment, 34 patients (17.3\%) had grade 3 elevations of bilirubin and $10(5.1 \%)$ had grade 3 decreases in serum albumin. Predictors of grade 3 toxicity in HCC patients included extrahepatic disease at the time of therapy $(t=2.2$, $p=0.03$ ). When evaluating individual measures of liver function, baseline liver function abnormality predicted both bilirubin $(t=3.7, p=0.0002)$ and albumin $(t=16.6$, $p<0.0001)$ grade 3 toxicities. Whole liver therapy predicted grade 3 bilirubin toxicity $(t=2, p=0.05)$ and increased baseline body mass index predicted grade 3 albumin toxicity $(t=2.79, p=0.006)$. The authors suggested that future trials focus on treatment-naive patients, as grade 3 toxicities in the SARAH and SIRveNIB trials were 3\% for grade 3 hyperbilirubinemia and $0.8 \%$ for grade 3 hypoalbuminemia. ${ }^{15,16} \mathrm{Com}-$ bining sorafenib with ${ }^{90} \mathrm{Y}$ may have significant additional risk. In the SORAMIC trial, Ricke et al reported an increase of grade 3 hyperbilirubinemia from 4.4 to $14.5 \%$ when sorafenib alone was used compared to sorafenib and ${ }^{90} \mathrm{Y} .{ }^{17}$

A large retrospective study by the European Network on Radioembolization with Yttrium-90 resin microspheres 

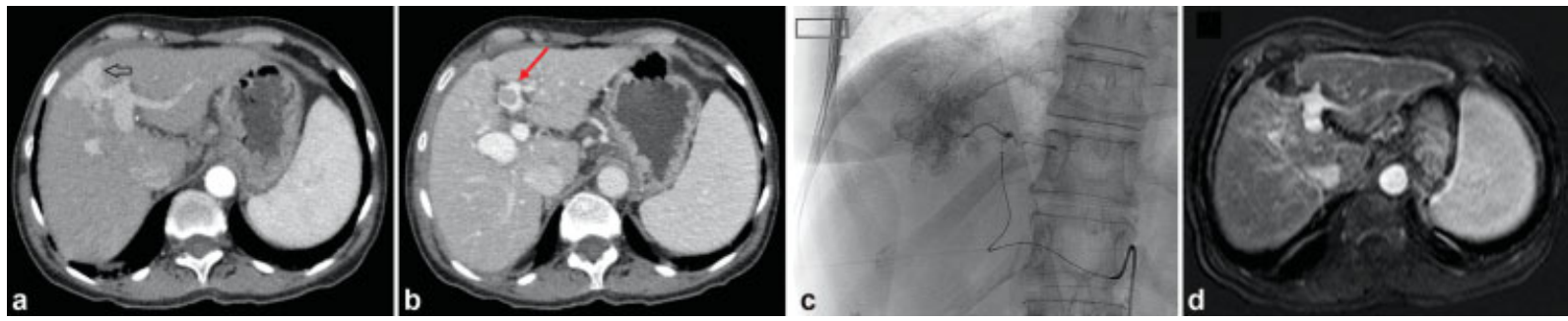

Fig. $2{ }^{90} \mathrm{Y}$ radioembolization in a patient with segmental portal vein thrombus. Child-Pugh A6 patient with postcontrast axial images (a, b) demonstrating a 3.2 enhancing hepatocellular carcinoma in segment IV (black arrow) with associated left segmental branch portal vein thrombus (red arrow). Segmental radioembolization (c) ultimately led to tumor necrosis on early arterial phase postcontrast MRI (d) with retraction of the venous thrombus.

group (ENRY) compared the use of radioembolization in 128 elderly ( $>70$ years old) and 197 younger ( $<70$ years old) patients who had similar demographic characteristics and found it to be equally well-tolerated and effective in both cohorts with predominantly grade 1 to 2 adverse events that were of short duration. ${ }^{23}$ There was no significant difference in median survival between the two groups $(p=0.942)$ in patients with early, intermediate, or advanced BCLC stage disease.

Patients with PVT are optimally treated with nonembolic therapy to avoid ischemic hepatitis, making ${ }^{90} \mathrm{Y}$ particularly useful for this indication. Iñarrairaegui et al reported findings in 25 patients with PVT treated with radioembolization. There were no grade 3 liver toxicities up to 2 months after treatment with median OS of 10 months. ${ }^{24}$ Kulik et al reported outcomes in 34 patients with PVT treated with glass microspheres. ${ }^{25}$ Patients tolerated treatment, but extension of branch PVT into the main portal vein was associated with a lower survival compared to segmental venous invasion (467 vs. 133.5 days, $p=0.0052$; - Fig. 2).

One other risk of radioembolization includes the development of radioembolization-induced liver disease (REILD), which is defined as jaundice or ascites appearing up to 3 months following ${ }^{90} \mathrm{Y}$ without tumor progression or bile duct occlusion. Zimmerman et al demonstrated that radioembolization with resin is safe in patients with history of prior major hepatic resection with none of the 15 studied patients developing REILD. ${ }^{26}$ In the study, the minimum time between surgery and radioembolization was 4 months (mean of 18 months), suggesting that a 4-month interval results in sufficient hypertrophy of the liver remnant to avoid REILD when dosing ${ }^{90} \mathrm{Y}$ (- Fig. 3). Maximizing delivery to tumor is important to avoid damage to normal tissue in the treatment zone. As tumor to normal ratio at mapping decreases, the risk of radiation damage due to nontarget delivery can increase. Allimant et al found that patients with higher dose deposition in the nontumoral liver following radioembolization were more likely to develop REILD $(p=0.04) .{ }^{10}$ In their study, nontumoral liver deposition was more than $58 \mathrm{~Gy}$ in all patients who developed REILD. Using external beam radiation as a reference, $55 \mathrm{~Gy}$ is the dose limit for treatment of one-third of the liver and $45 \mathrm{~Gy}$ is the limit for two-thirds of the total liver. ${ }^{27}$ In patients with limited tumor perfusion at mapping, dose reduction may potentially be required to limit toxicity in patients undergoing lobar or whole-liver treatment.

Pulmonary toxicity is directly related to absorbed dose. Therefore, radioembolization can be safe to use in patients
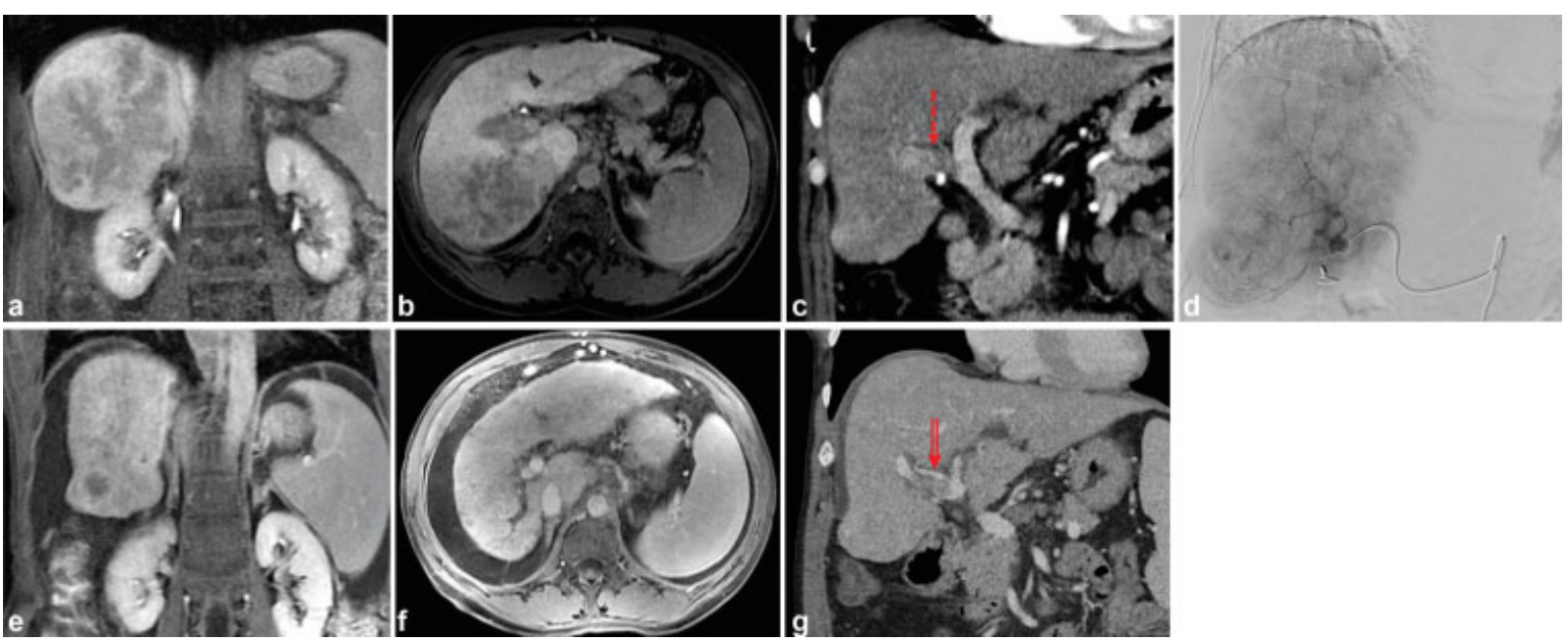

Fig. $3{ }^{90}$ Y radioembolization for a large mass with portal vein thrombosis (PVT). Child-Pugh A6 patient with a large posterior right hepatic mass seen on delayed phase contrast MRI (a, b) with a right lobar PVT shown on coronal postcontrast CT (c; dashed arrow). The patient was treated with lobar radioembolization (d). Follow-up coronal (e) and axial (f) MRI (coronal (e) demonstrates marked reduction in tumor size. The patient did develop new onset ascites. There was also decrease in PVT burden on CT (g, double arrow) along with the tumor response. 

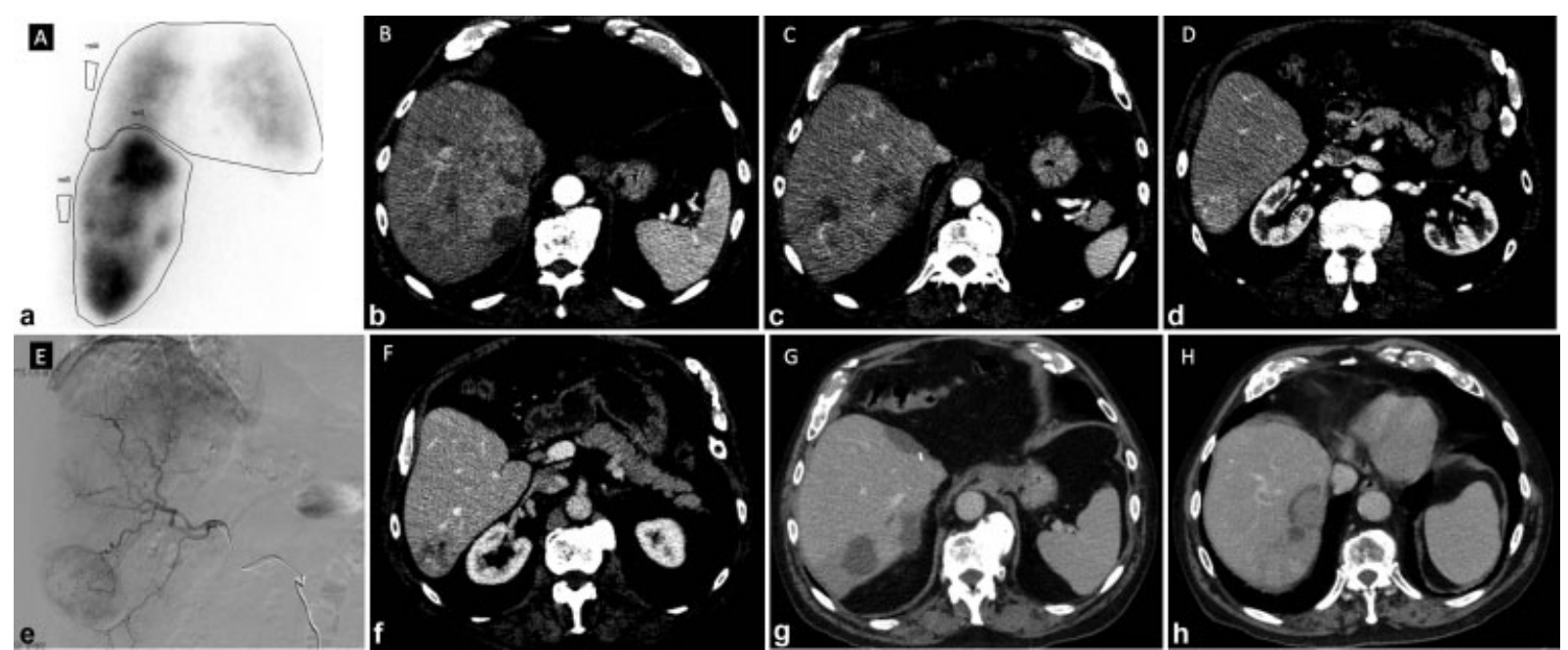

Fig. $4{ }^{90} \mathrm{Y}$ radioembolization in high lung shunt fraction. Child-Pugh A patient with multifocal recurrence in the right hepatic lobe (BCLC B) (a-d) following left lobectomy to attempt cure. He had a lung shunt fraction of $24 \%$ (a) on macroaggregated albumin shunt imaging. The calculated dose to his lungs even with this shunt was less than $30 \mathrm{~Gy}$. Given that the entire remaining liver was being treated, he was treated in two separate treatments (e) with half the total activity given at each visit. He developed no pulmonary symptoms. Postcontrast axial CT demonstrates significant cystic and hemorrhagic changes consistent with tumor necrosis $(\mathbf{f}-\mathbf{h})$.

with a lung shunt fraction greater than 10 to $15 \%$. Das et al performed ${ }^{90} \mathrm{Y}$ therapy in 103 patients with a mean lung shunt fraction of $24.4 \%$ (18.1-28.8\%). Twenty (19\%) of the patients developed nonspecific pulmonary symptoms including cough, shortness of breath, and wheezing within 1 year post ${ }^{90} \mathrm{Y}$ therapy with a median time to development of 63 days (range: $7-224) .{ }^{28}$ Thoracic imaging demonstrated no evidence of pulmonary injury or fibrosis following treatment, and there was no difference in the survival between patients with and without pulmonary symptoms (6.7 vs. 7.3 months, $p=0.903$ ). Based on their results, Das et al recommended keeping single-session lung dose below 25 Gray $^{28}$ (-Fig. 4).

While ${ }^{90} Y$ has been shown to be safe to use in the abovementioned groups, there are still methods that can improve safety such as staged therapy. Seidensticker et al found that sequential lobar radioembolization in noncirrhotic patients resulted in less hepatotoxicity compared to whole liver embolization. ${ }^{29}$ They found compensatory hypertrophy of the subsequently treated lobe in the sequential cohort compared to no volume changes following whole liver therapy. This compensatory hypertrophy may explain the increased liver tolerance in the sequentially treated cohort.

\section{Conclusion}

This review article summarizes the use of ${ }^{90} \mathrm{Y}$ therapy in intermediate- and advanced-stage (BCLC B and C) patients. Radioembolization is well-tolerated, safe, and efficacious in patients with normal liver function. Ideally, patients are treatment naive as well. ${ }^{90} \mathrm{Y}$ can be performed safely in both young and elderly patients and in selected patients with extensive disease, portal vein invasion, large hepatopulmonary shunt fractions, and in the setting of prior liver resection. ${ }^{90} \mathrm{Y}$ therapy is currently being studied in combination with current first-line systemic agents and with new immune modulators such as nivolumab and pembrolizumab. Continued evaluations may potentially improve the efficacy and safety of radioembolization therapy for HCC that has already been demonstrated.

\section{References}

1 Villanueva A. Hepatocellular carcinoma. N Engl J Med 2019;380 (15):1450-1462

2 Forner A, Reig M, Bruix J. Hepatocellular carcinoma. Lancet 2018; 391(10127):1301-1314

3 Salem R, Miller FH, Yaghmai V, Lewandowski RJ. Response assessment methodologies in hepatocellular carcinoma: complexities in the era of local and systemic treatments. J Hepatol 2013;58 (06):1260-1262

4 Salem R, Gilbertsen M, Butt Z, et al. Increased quality of life among hepatocellular carcinoma patients treated with radioembolization, compared with chemoembolization. Clin Gastroenterol Hepatol 2013;11(10):1358-1365.e1

5 Salem R, Gabr A, Riaz A, et al. Institutional decision to adopt Y90 as primary treatment for hepatocellular carcinoma informed by a 1,000-patient 15-year experience. Hepatology 2018;68(04): 1429-1440

6 Mantry PS, Mehta A, Madani B, Mejia A, Shahin I. Selective internal radiation therapy using yttrium-90 resin microspheres in patients with unresectable hepatocellular carcinoma: a retrospective study. J Gastrointest Oncol 2017;8(05):799-807

7 Ali R, Gabr A, Abouchaleh N, et al. Survival analysis of advanced HCC treated with radioembolization: comparing impact of clinical performance status versus vascular invasion/metastases. Cardiovasc Intervent Radiol 2018;41(02):260-269

8 Frantz S, Matsuoka L, Vaheesan K, et al. Multi-center evaluation of survival and toxicities following radioembolization of hepatocellular carcinoma: analysis of the RESiN Registry. J Vasc Interv Radiol 2021;32(06):845-852

9 Mazzaferro V, Sposito C, Bhoori S, et al. Yttrium-90 radioembolization for intermediate-advanced hepatocellular carcinoma: a phase 2 study. Hepatology 2013;57(05):1826-1837

10 Allimant C, Kafrouni M, Delicque J, et al. Tumor targeting and three-dimensional voxel-based dosimetry to predict tumor response, toxicity, and survival after yttrium-90 resin microsphere 
radioembolization in hepatocellular carcinoma. J Vasc Interv Radiol 2018;29(12):1662-1670.e4

11 Garin E, Tselikas L, Guiu B, et al; DOSISPHERE-01 Study Group. Personalised versus standard dosimetry approach of selective internal radiation therapy in patients with locally advanced hepatocellular carcinoma (DOSISPHERE-01): a randomised, multicentre, open-label phase 2 trial. Lancet Gastroenterol Hepatol 2021;6(01):17-29

12 Salem R, Gordon AC, Mouli S, et al. Y90 radioembolization significantly prolongs time to progression compared with chemoembolization in patients with hepatocellular carcinoma. Gastroenterology 2016;151(06):1155-1163.e2

13 Salem R, Lewandowski RJ, Kulik L, et al. Radioembolization results in longer time-to-progression and reduced toxicity compared with chemoembolization in patients with hepatocellular carcinoma. Gastroenterology 2011;140(02):497-507.e2

14 Zhang Y, Li Y, Ji H, Zhao X, Lu H. Transarterial Y90 radioembolization versus chemoembolization for patients with hepatocellular carcinoma: a meta-analysis. Biosci Trends 2015;9(05):289-298

15 Vilgrain V, Pereira H, Assenat E, et al; SARAH Trial Group. Efficacy and safety of selective internal radiotherapy with yttrium-90 resin microspheres compared with sorafenib in locally advanced and inoperable hepatocellular carcinoma (SARAH): an open-label randomised controlled phase 3 trial. Lancet Oncol 2017;18(12): 1624-1636

16 Chow PKH, Gandhi M, Tan SB, et al; Asia-Pacific Hepatocellular Carcinoma Trials Group. SIRveNIB: selective internal radiation therapy versus sorafenib in Asia-Pacific patients with hepatocellular carcinoma. J Clin Oncol 2018;36(19):1913-1921

17 Ricke J, Bulla K, Kolligs F, et al; SORAMIC Study Group. Safety and toxicity of radioembolization plus Sorafenib in advanced hepatocellular carcinoma: analysis of the European multicentre trial SORAMIC. Liver Int 2015;35(02):620-626

18 Javan H, Dayyani F, Abi-Jaoudeh N. Therapy in advanced hepatocellular carcinoma. Semin Intervent Radiol 2020;37(05):466-474

19 Zhan C, Ruohoniemi D, Shanbhogue KP, et al. Safety of combined yttrium-90 radioembolization and immune checkpoint inhibitor immunotherapy for hepatocellular carcinoma. J Vasc Interv Radiol 2020;31(01):25-34
20 El-Khoueiry AB, Sangro B, Yau T, et al. Nivolumab in patients with advanced hepatocellular carcinoma (CheckMate 040): an openlabel, non-comparative, phase $1 / 2$ dose escalation and expansion trial. Lancet 2017;389(10088):2492-2502

21 Zhu AX, Finn RS, Edeline J, et al; KEYNOTE-224 Investigators. Pembrolizumab in patients with advanced hepatocellular carcinoma previously treated with sorafenib (KEYNOTE-224): a nonrandomised, open-label phase 2 trial. Lancet Oncol 2018;19(07): 940-952

22 Brown D, Krebs $\mathrm{H}$, Brower J, et al. Incidence and risk factors for sustained hepatic function toxicity 6 months after radioembolization: analysis of the radiation-emitting sir-spheres in nonresectable liver tumor (RESIN) registry. J Gastrointest Oncol 2021;12(02):639-657

23 Golfieri R, Bilbao JI, Carpanese L, et al; European Network on Radioembolization with Yttrium-90 Microspheres (ENRY) study collaborators. Comparison of the survival and tolerability of radioembolization in elderly vs. younger patients with unresectable hepatocellular carcinoma. J Hepatol 2013;59(04):753-761

24 Iñarrairaegui M, Thurston KG, Bilbao JI, et al. Radioembolization with use of yttrium-90 resin microspheres in patients with hepatocellular carcinoma and portal vein thrombosis. J Vasc Interv Radiol 2010;21(08):1205-1212

25 Kulik LM, Carr BI, Mulcahy MF, et al. Safety and efficacy of 90Y radiotherapy for hepatocellular carcinoma with and without portal vein thrombosis. Hepatology 2008;47(01):71-81

26 Zimmermann M, Schulze-Hagen M, Liebl M, et al. Safety and efficacy of $\mathrm{y}-90$ radioembolization after prior major hepatic resection. Cardiovasc Intervent Radiol 2017;40(08):1206-1212

27 Emami B, Lyman J, Brown A, et al. Tolerance of normal tissue to therapeutic irradiation. Int J Radiat Oncol Biol Phys 1991;21(01):109-122

28 Das A, Riaz A, Gabr A, et al. Safety and efficacy of radioembolization with glass microspheres in hepatocellular carcinoma patients with elevated lung shunt fraction: analysis of a 103patient cohort. Eur J Nucl Med Mol Imaging 2020;47(04):807-815

29 Seidensticker R, Seidensticker M, Damm R, et al. Hepatic toxicity after radioembolization of the liver using (90)Y-microspheres: sequential lobar versus whole liver approach. Cardiovasc Intervent Radiol 2012;35(05):1109-1118 